\title{
Light hadron spectroscopy at BESIII
}

\section{Giulio Mezzadri*†}

Università di Ferrara (IT), INFN Sezione di Ferrara (IT)

E-mail: giulio.mezzadri@fe.infn.it

The BESIII experiment has accumulated a large sample of $J / \psi, \psi^{\prime}$ and $\psi(3770)$ data set. Through these charmonium radiative and hadronic decays, we can explore the light hadron spectroscopies. In this talk, we will report our recent results on the properties of the $\mathrm{X}$ states, such as $\mathrm{X}(1835)$ and $\mathrm{X}(p \bar{p})$, study of $J / \psi \rightarrow$ radiative decays $\left(J / \psi \rightarrow \gamma K_{S} K_{s} \eta, \gamma \pi^{+} \pi^{-} \eta^{\prime}, \gamma \pi^{0} \pi^{0}\right)$ and progress on the baryon spectroscopy ( $\Xi^{*}$ states other related topics on baryonic pair production). In addition, we also present our results on the decays of light mesons $\eta$ and $\eta^{\prime}$ at BESIII

The European Physical Society Conference on High Energy Physics 22-29 July 2015

Vienna, Austria

*Speaker.

${ }^{\dagger}$ On behalf of the BESIII Collaboration. 


\section{Introduction}

One of the main open questions of the high energy physics is related to the understanding of the energy region below the $J / \psi$. There, the theorems of the perturbative Quantum ChromoDynamics (pQCD) are not anymore reliable and it is missing one theory that can give quantitative predictions on different processes. Up to now, different models, like Vector Meson Dominance (VMD) [1], are good attempts to answer these questions, but no one seems to have a complete understanding of the whole problematics.

The study of the light hadron spectroscopy opens a way to start understanding the behaviour and the recurrencies in these states and may be an useful proof to shed light in the plethora of different theoretical predictions. With its unprecedents statistic collected at $J / \psi, \psi^{\prime}$ and $\psi(3770)$, the BESIII experiment [2] has the opportunity to test different theoretical models studying radiative and hadronic decays of charmonia to these light states and to point out interesting properties typical of this energy regime. At the same time, because of the largest statistic in the world, it can search for new unpredicted new states and for decay modes theoretically predicted but never discovered.

BEPCII is a double-ring multi-bunch $e^{+} e^{-}$collider running in the tau-charm energy region The BESIII detector, described in detail in [2], has a geometrical acceptance of $93 \%$ of $4 \pi$ solid angle. It consist of a drift chamber (MDC), a time-of-flight (TOF) system, and an electromagnetic calorimeter (EMC), all enclosed in a super-conductiong solenoid with $1.0 \mathrm{~T}$ magnetic field.

In this work, the attention will be focused on two of the latest results of BESIII: the analysis of the properties of $X(18 X X)$ states, a series of new pseudoscalar states close to the proton-antiproton threshold mass, with a focus on the $J / \psi \rightarrow \gamma K_{S}^{0} K_{S}^{0} \eta$ partial wave analysis [3]; the first observation of the Dalitz decay of $\eta \rightarrow \gamma e^{+} e^{-}$, using a radiative decay of $J / \psi$ [4], proving that BESIII can be also considered a proper $\eta-$ factory.

\section{Review of the status of BESIII X(18XX) states}

The X(1835) was first discovered by BESII in the $J / \psi$ radiative decay channel $J / \psi \rightarrow \gamma \eta^{\prime} \pi^{+} \pi^{-}$ [5] and later confirmed by BESIII in the same channel [6]. Moreover, a plethora of new states were observed in the same mass region in different radiative decay mode of $J / \psi$ ([7], [8], [9]). A structure in the $J / \psi$ radiative decay in proton-antiproton pair was also observed [10], with a peak nearby the $\mathrm{X}(1835)$ mass.

Different hypothesis about its nature were made: theoretician pointed out that the state might be the $\eta^{\prime}$ second radial excitation [11], a $p \bar{p}$ bound state [12] or a glueball [13]. It is necessary to measure its $J^{P C}$ and to search new decay modes. In the following lines is reported the first observation and spin-parity determination of the $\mathrm{X}(1835)$ in $J / \psi \rightarrow \gamma K_{S}^{0} K_{S}^{0} \eta$ by means of a Partial Wave Analysis (PWA). The analysis is based on a sample of $(1310.6 \pm 10.5) \times 10^{6} \mathrm{~J} / \psi$.

The invariant mass spectrum of $K_{S}^{0} K_{S}^{0} \eta$ is shown in Fig 1 (a). Besides a clear $\eta_{c}$ signal, a structure around $1.85 \mathrm{GeV} / \mathrm{c}^{2}$ is observed. Fig 1 (b) shows the $K_{S}^{0} K_{S}^{0}$ mass spectrum. It shows a strong enhancement near the threshold, which is interpreted as the $f_{0}(980)$ by considering spinparity and isospin conservation. The scatter plot of the invariant mass of $K_{S}^{0} K_{S}^{0}$ versus that of $K_{S}^{0} K_{S}^{0} \eta$ is shown in Fig 1 (c). A clear accumulation of events is seen around the intersection of the $f_{0}(980)$ and the structure arounf $1.85 \mathrm{GeV} / c^{2}$. The correletion between the structure of 1.85 
$\mathrm{GeV} / c^{2}$ and the $f_{0}(980)$ is made more clear, by requiring $M_{K_{S}^{0} K_{S}^{0}}<1.1 \mathrm{GeV} / \mathrm{c}^{2}$ : in this way, the structure becomes much more prominent, as shown in Fig 1 (d).
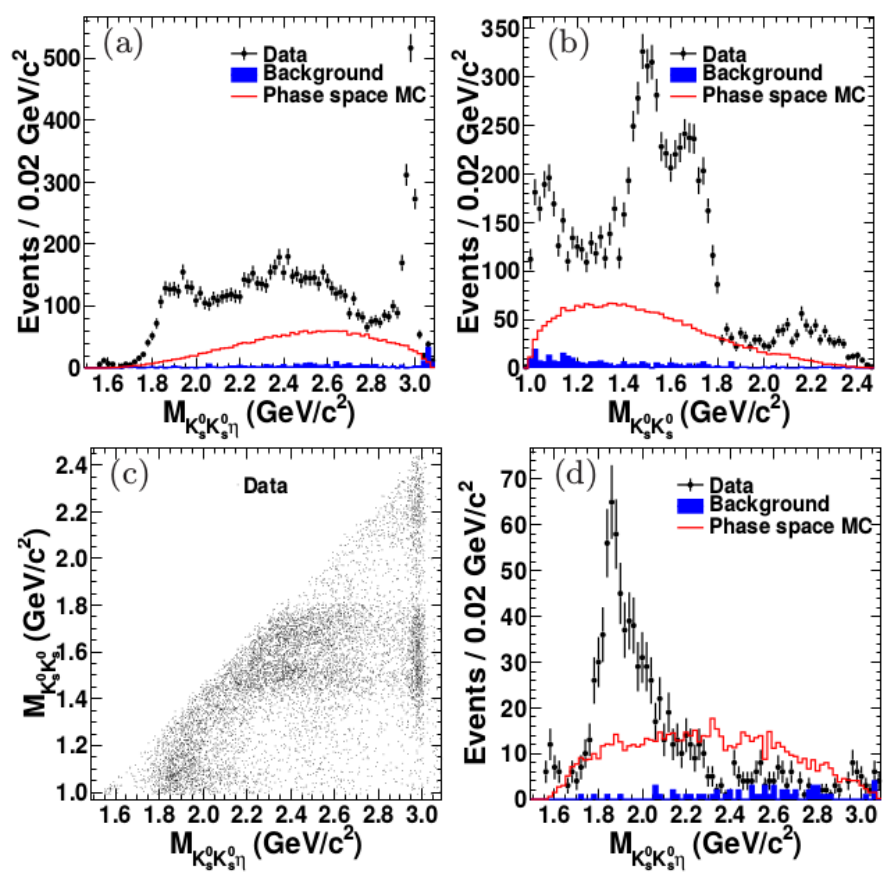

Figure 1: Invariant mass distribution for selected events: (a) and (b) are the invariant mass spectra of $K_{s}^{0} K_{s}^{0} \eta$ and $K_{s}^{0} K_{s}^{0}$, respectively; (c) is the scatter plot of the invariant mass of $K_{s}^{0} K_{s}^{0}$ versus that of $K_{s}^{0} K_{s}^{0} \eta$; (d) is the $K_{s}^{0} K_{s}^{0} \eta$ invariant mass spectrum for events with the requirement $M_{K_{S}^{0} K_{S}^{0}}<1.1 \mathrm{GeV} / c^{2}$. The dots with error bars are data; the shaded histograms are the non- $\eta$ backgrounds estimated by the $\eta$ sideband; the solid histogrmas are phase space MC events of $J / \psi \rightarrow \gamma K_{s}^{0} K_{s}^{0} \eta$ passing the same event selction and with aribitrary normalization.

A PWA of events satisfying $M_{K_{S}^{0} K_{S}^{0} \eta}<2.8 \mathrm{GeV} / \mathrm{c}^{2}$ and $M_{K_{S}^{0} K_{S}^{0}}<1.1 \mathrm{GeV} / \mathrm{c}^{2}$ is performed to determine the parameters of the structure. These restrictions reduce complexities due to intermediate processes. Parity conservation in the $J / \psi \rightarrow \gamma K_{S}^{0} K_{S}^{0} \eta$ decay restricts the possible $J^{P C}$ of the $K_{S}^{0} K_{S}^{0} \eta(\mathrm{X})$ system to be $0^{-+}, 1^{++}, 2^{++}, 2^{-+}, 3^{++}$, etc. Only spins $J<3$ and possible $S$-wave or $P$-wave decays of the $\mathrm{X}$ are considered.

The $K_{S}^{0} K_{S}^{0} \eta, K_{S}^{0} K_{S}^{0}, K_{S}^{0} \eta$ mass spectra are shown in Fig. 2. Overlaid on the data are the PWA fit projections, as well as the individual contributions from each component. The spin-parity of the $\mathrm{X}(1835)$ is determined to be $0^{-+}$and the mass and width of the $\mathrm{X}(1835)$ are measured to be $1844 \pm 9(\text { stat })_{-25}^{+16}$ (syst) $\mathrm{MeV} / \mathrm{c}^{2}$ and $192_{-17}^{+20}(\text { stat })_{-43}^{+62}$ (syst) $\mathrm{MeV}$, respectively. The mass and the width of the $\mathrm{X}(1835)$ are consistent with the values obtained from the decay $J / \psi \rightarrow \gamma \pi^{+} \pi^{-} \eta^{\prime}$ by BESIII. These results are all first-time measurements and provide important information to further understand the nature of $\mathrm{X}(1835)$. Another $0^{-+}$state, the $\mathrm{X}(1560)$, is observed in data and is seen to interfere with the $\mathrm{X}(1835)$. Present statistics do not allow to conclusively determine if the $\mathrm{X}(1560)$ is the same state as $\eta(1405) / \eta(1475)$. 

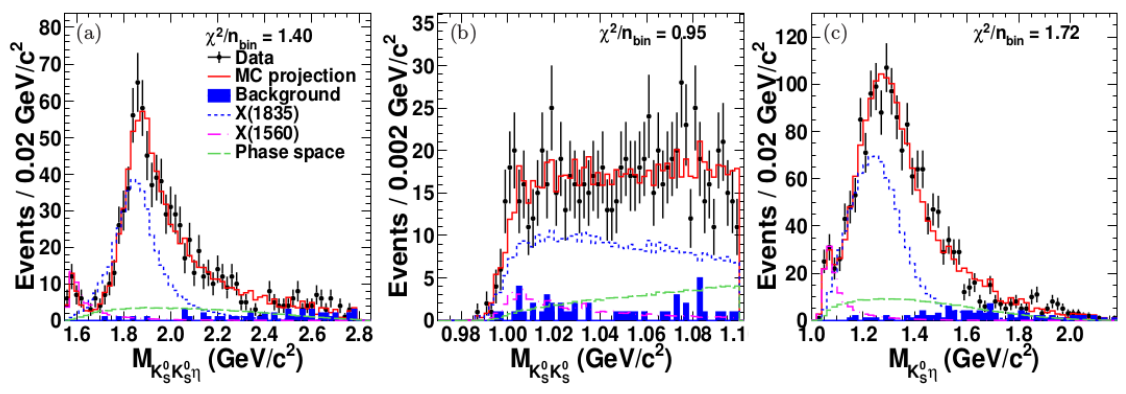

Figure 2: Comparison between data and PWA fit projections. (a), (b) and (c) are the invariant mass distribution of $K_{S}^{0} K_{S}^{0} \eta, K_{S}^{0} K_{S}^{0}$, and $K_{S}^{0} \eta$ (two entries/event), respectively. The dots with error bars are data, the solid histograms are the PWA total projections, and the short-dashed, dash-dotted and long-dashed histograms show the contribution of $\mathrm{X}(1835), \mathrm{X}(1560)$ and the non-resonant component, respectively.

\section{Observation of Dalitz decay $\eta \rightarrow \gamma e^{+} e^{-}$}

Electromagnetic (EM) Dalitz decays of light pseudoscalar mesons, $P \rightarrow \gamma l^{+} l^{-}\left(P=\pi^{0}, \eta, \eta^{\prime}\right.$; $l=e, \mu)$, play an important role in revealing the structure of hadrons and the interaction mechanism between photons and hadron [14]. If one assume point-like particles, the decay rates can be exactly calculated by Quantum ElectroDynamics (QED) [15]. Modifications to the QED decay rate due to the inner structure of the mesons are encoded in the transition form factor (TFF) $F\left(q^{2}\right)$, where $q$ is the momentum transferred to the lepton pair, and $q^{2}$ is the square of the invariant mass of the lepton pair.

In the following lines, the first observation of the Dalitz decay $\eta^{\prime} \rightarrow \gamma e^{+} e^{-}$is presented. The differential decay width, normalized to the radiative decay width $\eta^{\prime} \rightarrow \gamma \gamma$, is

$$
\begin{aligned}
& \frac{d \Gamma\left(\eta^{\prime} \rightarrow \gamma e^{+} e^{-}\right)}{d q^{2} \Gamma\left(\eta^{\prime} \rightarrow \gamma \gamma\right)}=\frac{2 \alpha}{3 \pi} \frac{1}{q^{2}} \sqrt{1-\frac{4 m_{l}^{2}}{q^{2}}}\left(1+\frac{2 m_{l}^{2}}{q^{2}}\right)\left(1-\frac{q^{2}}{m_{\eta^{\prime}}^{2}}\right)^{3}\left|F\left(q^{2}\right)\right|^{2} \\
& =\left[Q E D\left(q^{2}\right)\right] \times\left|F\left(q^{2}\right)\right|^{2},
\end{aligned}
$$

where $m_{\eta^{\prime}}$ and $m_{l}$ are the masses of the $\eta^{\prime}$ meson and the lepton respectively; $\alpha$ is the fine structure constant; and $\left[Q E D\left(q^{2}\right)\right]$ represents the calculable QED part for a point-like meson. $F\left(q^{2}\right)$ can be experimentally determined from differences between the measured di-lepton invariant mass spectrum and the QED calculation. In the VMD model [1] it is assumed that interaction between virtual photon and hadrons are dominated by a superposition of neutral vector meson states. The parameter to be experimentally determined is the slop of the form factor $b$, which is related to the effective virtual vector meson mass $\Lambda$ by

$$
b=\left(\frac{d F}{d q^{2}}\right)_{q^{2}=0}=\Lambda^{-2}
$$

In experiments, the single-pole form factor is generally used to extract the slope of the form factor. For the case of $\eta^{\prime}$, the pole is expected to lie within the kinematic boundaries of the decay. 
The square of the form factor is described by

$$
\left|F\left(q^{2}\right)\right|^{2}=\frac{\Lambda^{2}\left(\Lambda^{2}+\gamma^{2}\right)}{\left(\Lambda^{2}-q^{2}\right)^{2}+\Lambda^{2} \gamma^{2}}
$$

where $\Lambda$ and $\gamma$ correspond to the mass and the width of the Breit-Wigner shape for the effective contribuiting vector meson.

For the $\eta^{\prime}$ Dalitz decay, only the process $\eta^{\prime} \rightarrow \gamma \mu+\mu$ - has been observed and the slope of the form factor was measured to be $b_{\eta^{\prime}}=(1.7 \pm 0.4) \mathrm{GeV}^{-2}$ [14], [16] . In the VMD model, the TFF slope is expected to be $b_{\eta^{\prime}}=(1.45) \mathrm{GeV}^{-2}$ [17], while for ChPT it is $b_{\eta^{\prime}}=(1.60) \mathrm{GeV}^{-2}$ [18].

After that selection criteria were applied for both the signal and normalization channels, the ratio $\Gamma\left(\eta^{\prime} \rightarrow \gamma e^{+} e^{-}\right) / \Gamma\left(\eta^{\prime} \rightarrow \gamma \gamma\right)$ is determined using the following formula:

$$
\frac{\Gamma\left(\eta^{\prime} \rightarrow \gamma e^{+} e^{-}\right)}{\Gamma\left(\eta^{\prime} \rightarrow \gamma \gamma\right)}=\frac{N_{\eta^{\prime} \rightarrow \gamma e^{+} e^{-}}}{N_{\eta^{\prime} \rightarrow \gamma \gamma}} \cdot \frac{\varepsilon_{\eta^{\prime} \rightarrow \gamma \gamma}}{\varepsilon_{\eta^{\prime} \rightarrow \gamma e^{+} e^{-}}}
$$

where $N_{\eta^{\prime} \rightarrow \gamma e^{+} e^{-}}\left(N_{\eta^{\prime} \rightarrow \gamma \gamma}\right)$ and $\varepsilon_{\eta^{\prime} \rightarrow \gamma \gamma}\left(\varepsilon_{\eta^{\prime} \rightarrow \gamma e^{+} e^{-}}\right)$are the number of observed signal events and the detection efficiency, respectively. The results is

$$
\frac{\Gamma\left(\eta^{\prime} \rightarrow \gamma e^{+} e^{-}\right)}{\Gamma\left(\eta^{\prime} \rightarrow \gamma \gamma\right)}=(2.13 \pm 0.09(\text { stat }) \pm 0.07(\text { sys })) \times 10^{-2} .
$$

The TFF is extracted from the bin-by-bin efficiency corrected signal yields for eight different $M\left(e^{+} e^{-}\right)$bins. The bin widths are all chosen to be $0.1 \mathrm{GeV} / \mathrm{c}^{2}$. Figure 3 shows the efficiency corrected signal yield versus $M\left(e^{+} e^{-}\right)$with the QED shape superimposed for comparison. The discrepancy between QED and data, which reflects the TFF, is evident in the high $M\left(e^{+} e^{-}\right)$. From the fit of the ratio of each bin yield with respect to the QED predication in each $M\left(e^{+} e^{-}\right)$interval, shown in Fig. 4, is possible to extract the slope of the form factor. A value of $b_{\eta^{\prime}}=(1.60 \pm$ $0.19) \mathrm{GeV}^{-2}$ is obtained, in agreement with the value in the process $\eta^{\prime} \rightarrow \gamma \mu^{+} \mu^{-}$and within errors with the VMD model predictions.

\section{References}

[1] J.J. Sakurai, Currents and Mesons, Ann. of Physics, 11 (1960).

[2] M. Ablikim et al. (BESIII Collaboration), Nucl. Instrum. Meth. A 614, 345 (2010).

[3] arXiv:1506.04807

[4] M. Ablikim et al. (BESIII Collaboration), Phys. Rev. D 64, 012001 (2015).

[5] M. Ablikim et al. (BES Collaboration), Phys. Rev. Lett. 95, 262001, (2005).

[6] M. Ablikim et al. (BESIII Collaboration), Phys. Rev. Lett. 106, 072002 (2011).

[7] M. Ablikim et al. (BESIII Collaboration), Phys. Rev. D 88, 091502.

[8] M. Ablikim et al. (BESIII Collaboration), Phys. Rev. Lett. 107182001.

[9] M. Ablikim et al. (BESIII Collaboration), Phys. Rev. D 87, 032008.

[10] M. Ablikim et al. (BESIII Collaboration), Phys. Rev. Lett. 108, 112003. 


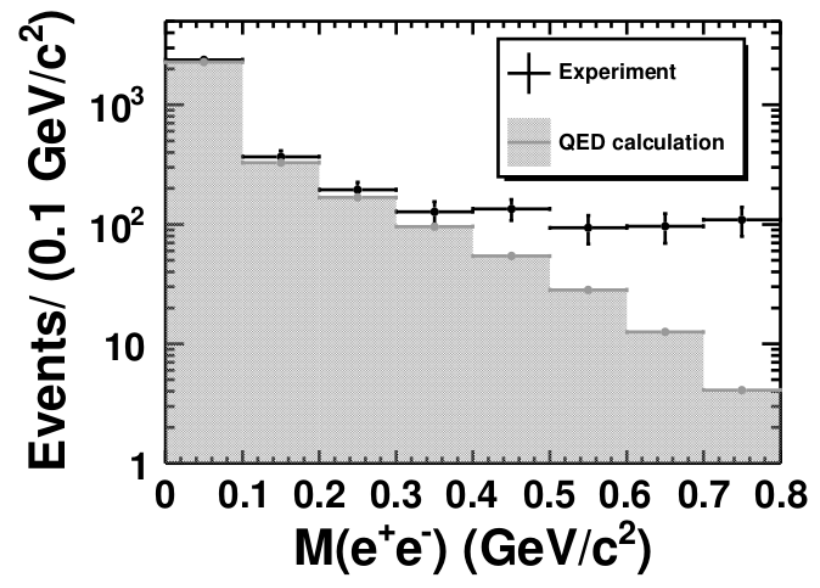

Figure 3: Efficiency-corrected signal yield $n_{i}^{\text {corr }}$ versus $M\left(e^{+} e^{-}\right)$. The (black) crosses are data and the (gray) shaded histogram indicates the point-like QED results.

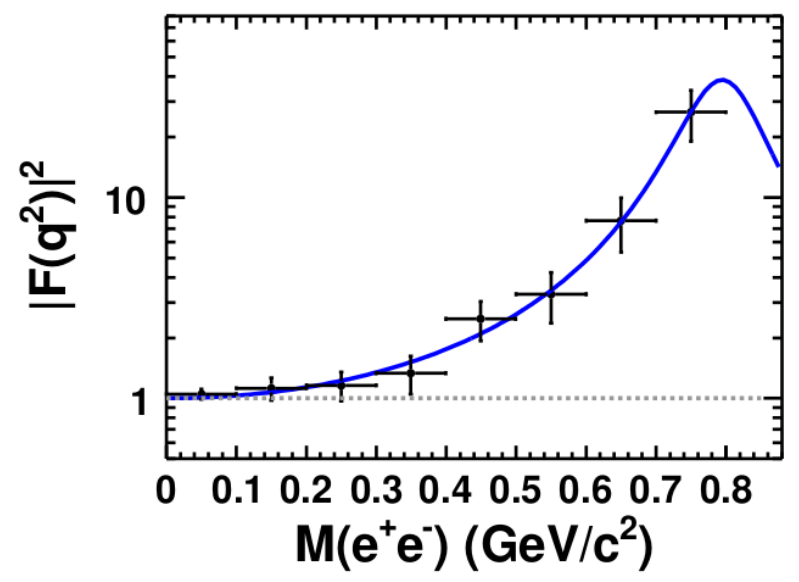

Figure 4: Fit to the single pole form factor $|F|^{2}$. The (black) crosses are data, where statistical and systematic uncertainties are combined, the (blue) solid curve shoes the fit results. The (gray) dotted line shows the point-like case (i.e. $|F|^{2}=1$ ) for comparison

[11] T. Huang, S.H. Zhu, Phys.Rev. D 73014023 (2006)

[12] Z.G. Wang, Chin. Phys. Lett. 27101201 (2010).

[13] B.A. Li, Phys. Rev. D 74034019 (2006)

[14] L.G. Landsberg, Phys. Rept. 128, 301 (1985).

[15] N.M. Krool, W. Wada, Phys. Rev. 98, 1355 (1955).

[16] R.I. Dzhelyadin et al., Phys. Lett. B 88379 (1979) [JETP Lett. 30, 359 (1979)].

[17] A, Bramon, E. Masso, Phys. Lett. B 104, 311 (1981).

[18] L. Ametller, L. Bergstrom, A. Bramon, E. Masso, Nucl. Phys. B 228, 301 (1983). 